$11-1-2010$

\title{
Adjusted Confidence Interval for the Population Median of the Exponential Distribution
}

Moustafa Omar Ahmed Abu-Shawiesh

Hashemite University, Zarqa Jordan, mabushawiesh@hu.edu.jo

Follow this and additional works at: http://digitalcommons.wayne.edu/jmasm

Part of the Applied Statistics Commons, Social and Behavioral Sciences Commons, and the Statistical Theory Commons

\section{Recommended Citation}

Abu-Shawiesh, Moustafa Omar Ahmed (2010) "Adjusted Confidence Interval for the Population Median of the Exponential Distribution," Journal of Modern Applied Statistical Methods: Vol. 9 : Iss. 2 , Article 13.

DOI: $10.22237 /$ jmasm/1288584720

Available at: http://digitalcommons.wayne.edu/jmasm/vol9/iss2/13

This Regular Article is brought to you for free and open access by the Open Access Journals at DigitalCommons@WayneState. It has been accepted for inclusion in Journal of Modern Applied Statistical Methods by an authorized editor of DigitalCommons@WayneState. 


\title{
Adjusted Confidence Interval for the Population Median of the Exponential Distribution
}

\author{
Moustafa Omar Ahmed Abu-Shawiesh \\ Hashemite University, \\ Zarqa Jordan
}

The median confidence interval is useful for one parameter families, such as the exponential distribution, and it may not need to be adjusted if censored observations are present. In this article, two estimators for the median of the exponential distribution, $M D$, are considered and compared based on the sample median and the maximum likelihood method. The first estimator is the sample median, $M D_{l}$, and the second estimator is the maximum likelihood estimator of the median, $M D_{M L E}$. Both estimators are used to propose a modified confidence interval for the population median of the exponential distribution, $M D$. Monte Carlo simulations were conducted to evaluate the performance of the proposed confidence intervals with respect to coverage probability, average width and standard error. A numerical example using a real data set is employed to illustrate the use of the modified confidence intervals; results are shown.

Key words: Exponential distribution, maximum likelihood estimator, sample median, confidence interval, coverage probability, average width.

Introduction

In most situations, researchers are interested in the estimate of the median of the population from which the sample data was drawn. Point estimates, such as the sample median, are of limited value because it is not possible to attach statements regarding the amount of confidence in their estimation of an unknown parameter. Of great value is an interval estimate, an estimate about which a researcher can make statements of confidence called the confidence interval (Daniel, 1990). A confidence interval provides much more information about the population value of the quantity of interest than does a point estimate (Smithson, 2001). Furthermore, the confidence intervals provide a way to report an estimate of a population parameter along with some information about the estimates precision. Although different settings lead to different formulas for computing confidence intervals, the

Moustafa Omar Ahmed Abu-Shawiesh is a member of the Faculty of Science in the Department of Mathematics. Email him at: mabushawiesh@hu.edu.jo. basic interpretation is always the same. A twosided confidence interval is the probability that a given parameter lies between a certain lower bound and upper bound (Kececioglu, 2002). According to Lewis (1996, page 216), confidence intervals are important because they are "the primary means by which the precision of a point estimator can be determined" and provide "lower and upper confidence limits to indicate how tightly the sampling distribution is compressed around the true value of the estimated quantity". The median confidence interval is useful for one parameter families, such as the exponential distribution, and it may not need to be adjusted if censored observations are present (Patel, et al., 1976).

The objective of this study is to modify the confidence interval for the population median of the exponential distribution, $M D$, based on two methods; the first method is based on the sample median, $M D_{l}$, while the second method is based on the maximum likelihood estimator of the median, $M D_{M L E}$. It is assumed that the underlying random sample $X_{1}, X_{2}, \ldots, X_{n}$ comes from an exponential distribution. The performance of the proposed 
modified confidence intervals is evaluated and compared using a Monte Carlo simulation to calculate the estimated coverage probability, the average width and the standard error; the use of these newly proposed methods is illustrated by a numerical example.

\section{The Exponential Distribution}

The exponential distribution is one of the most important and widely used continuous probability distributions in statistical practice. It possesses several important statistical properties, and yet exhibits great mathematical tractability (Balakrishnan \& Basu, 1996). It is the most frequently used distribution in such fields as queuing theory, reliability theory and reliability engineering where in this case it is provide models which are used to study many industrial phenomena such as time between machine breakdowns, length of queues or waiting time problems, at repair or processing facilities and the reliability of electronic systems, for example how long it takes for a bank teller to serve a customer (Maguire, et al., 1952; Betteley, et al., 1994 ; Montgomery, 2005). The exponential distribution also plays an important part in life testing problems; it would be an adequate choice for a situation where the failure rate appears to be more or less constant (Sinha \& Bhattacharjee, 2004). The exponential distribution may be viewed as a continuous counterpart of the geometric distribution, which describes the number of Bernoulli trials necessary for a discrete process to change state. In contrast, the exponential distribution describes the time for a continuous process to change state (Trivedi, 2001). Furthermore, the exponential distribution is related to Poisson in much the same way as the geometric is to binomial, where in a Poisson process the time between events has an exponential distribution (Betteley, et al., 1994). The exponential distribution is also the only continuous distribution having what is called the memoryless property, that is, the future lifetime of an individual has the same distribution no matter how it is at present.

The random variable $X$ has an exponential distribution with the rate parameter $\lambda$, that is, $X \sim \operatorname{Exp}(1 / \lambda)$, if and only if the density of it can be written as follows:

$$
\mathrm{f}(\mathrm{x} ; \lambda)=\lambda \mathrm{e}^{-\lambda \mathrm{x}}, \mathrm{x} \geq 0, \lambda>0
$$

The parameter $\lambda$, represents the mean number of events per unit time (e.g., the rate of arrivals or the rate of failure). The exponential distribution is supported on the interval $[0, \infty)$. The mean (expected value) of an exponentially distributed random variable $X$ with rate parameter $\lambda$ is given by:

$$
\mu=E(X)=\frac{1}{\lambda}
$$

In light of the examples given above, this makes sense: if a person receives phone calls at an average rate of 2 per hour, then they can expect to wait one-half hour for every call. Also, note that approximately $63 \%$ of the possible values lie below the mean for any exponential distribution (Betteley, et al., 1994).

The median of an exponentially distributed random variable $X$ with rate parameter $\lambda$ is given by:

$$
M D=\frac{\ln (2)}{\lambda}=\frac{0.69315}{\lambda}
$$

The maximum likelihood estimator (MLE) for the rate parameter $\lambda$, given an independent and identically distributed random sample of size $n$, $X_{1}, X_{2}, \ldots, X_{n}$, drawn from the exponential distribution, $\operatorname{Exp}(1 / \lambda)$, is given by:

$$
\hat{\lambda}=\frac{\mathrm{n}}{\sum_{\mathrm{i}=1}^{\mathrm{n}} \mathrm{X}_{\mathrm{i}}}=\frac{1}{\overline{\mathrm{X}}}
$$

While this estimate is the most likely reconstruction of the true parameter $\lambda$, it is only an estimate, and as such, the more data points available the better the estimate will be. Also, the MLEs are consistent estimators of their parameters and are asymptotically efficient (Casella \& Berger, 2002).

\section{The Used Estimators}

The sample mean, $\bar{X}$, and the 


\section{ABU-SHAWIESH M.O.A}

sample median, $M D_{1}$, which are used in this study for constructing the proposed modified confidence intervals for the exponential distribution median, $M D$, are now introduced.

\section{The Sample Mean, $\bar{X}$}

The sample mean is the most well known example of a measure of location, or average. It is defined for a set of values as the sum of values divided by the number of values and is denoted by $\bar{X}$. The sample mean for a random sample of size $n$ observations $X_{1}, X_{2}, \ldots, X_{n}$ can be defined as follows:

$$
\bar{X}=\frac{\sum_{i=1}^{n} X_{i}}{n}
$$

The main advantages of the sample mean, $\bar{X}$, are: it is easy to compute, easy to understood and takes all values into account. Its main disadvantages are: it is influenced by outliers, can be considered unrepresentative of data where outliers occur because many values may be well away from it and it requires all values in order to calculate its value (Betteley, et al., 1994; Francis, 1995).

\section{The Sample Median, $\mathrm{MD}_{1}$}

The sample median is perhaps the best known of the resistant location estimators. It is insensitive to behavior in the tails of the distribution. The sample median is defined for a set of values as the middle value when the values are arranged in order of magnitude and it is denoted herein by $M D_{1}$. The sample median for a random sample of size $n$ observations $X_{1}, X_{2}, \ldots, X_{n}$ can be defined as follows:

$$
M D_{1}= \begin{cases}X_{\left(\frac{n+1}{2}\right)} & \text { if } n \text { is odd } \\ \frac{X_{\left(\frac{n}{2}\right)}+X_{\left(\frac{n}{2}+1\right)}}{2} & \text { if } n \text { is even }\end{cases}
$$

The main advantages of the sample median, $M D_{l}$, is that, it is easy to determine, requires only the middle values to calculate, can be used when a distribution is skewed - as in the case of the exponential distribution, is not affected by outliers and has a maximal $50 \%$ breakdown point. The main disadvantages of the sample median, $M D_{l}$, are that it is difficult to handle in mathematical equations, it does not use all available values and it can be misleading in a distribution with a long tail because it discards so much information. The sample median, though, is considered as an alternative average to the sample mean (Betteley, et al., 1994; Francis, 1995). However, the sample median, $M D_{l}$, has become as a good general purpose estimator and is generally considered as an alternative average to the sample mean, $\bar{X}$.

Estimating the Exponential Distribution Median:

Two techniques are now introduced for finding estimates, the method of sample median and the method of maximum likelihood which is the most widely used.

\section{The Method of Sample Median}

Given a random sample of size $n$ observations, $X_{1}, X_{2}, \ldots, X_{n}$, the estimator of the exponential population median, $M D$, based on the method of sample median, $M D_{1}$, is denoted by $M D_{M D I}$. Now, from equation (3):

$$
\mathrm{MD}=\frac{1}{\lambda} \ln (2) \Rightarrow \lambda=\frac{\ln (2)}{\mathrm{MD}}
$$

Thus, if the exponential population median $M D$ in (8) is estimated by the sample median $M D_{l}$, results in the following approximation:

$$
\hat{\lambda}=\frac{\ln (2)}{M D_{1}} \text {. }
$$

Therefore, equating the results in (4) and (9) and solving, the following approximation is obtained: 


$$
\hat{\lambda}=\frac{n}{\sum_{i=1}^{n} X_{i}} \cong \frac{\ln (2)}{M D_{1}} \Rightarrow \sum_{i=1}^{n} X_{i} \cong \frac{n M D_{1}}{\ln (2)}
$$

The Maximum Likelihood Estimator of the Median

Given a random sample of size $n$ observations, $X_{1}, X_{2}, \ldots, X_{n}$, the estimator of the exponential population median based on the maximum likelihood method is denoted herein by $M D_{M L E}$ and can be defined as follows:

$$
M D_{M L E}=\frac{1}{\hat{\lambda}} \ln (2)=\frac{\sum_{i=1}^{n} X_{i}}{n} \ln (2)
$$

where

$$
\hat{\lambda}=\frac{\mathrm{n}}{\sum_{\mathrm{i}=1}^{\mathrm{n}} \mathrm{X}_{\mathrm{i}}}=\frac{1}{\overline{\mathrm{X}}}
$$

Comparing the Two Estimators of the Exponential Distribution Median

It is known that the maximum likelihood estimators are asymptotically unbiased and efficient. Concretely, the estimator $M D_{M L E}$ is unbiased and $\operatorname{Var}\left(M D_{M L E}\right)=\frac{\ln ^{2}(2)}{n \lambda^{2}}$. Moreover, the sample median estimator, $M D_{l}$, is asymptotically normal distributed with asymptotic variance $A \operatorname{Var}\left(M D_{1}\right)=\frac{1}{4 n f^{2}(M D)}$, where $f($.$) is$ the corresponding density and $M D$ is the theoretical median (Vann deer Vaart, 1998). Asymptotically unbiased means that the average value over many random samples for the two estimators $M D_{1}$ or $M D_{M L E}$ is the exponential distribution median, $M D$. To compare the two estimators $M D_{1}$ and $M D_{M L E}$ in terms of how far they are from the exponential distribution median $(M D)$ on the average for many random samples, it is necessary to compare their root mean square error, RMSE, given as follows:

$$
R M S E=\sqrt{\frac{1}{r} \sum_{i=1}^{r}\left(M D_{i}-M D\right)^{2}}
$$

where $M D_{1}, M D_{2}, \ldots, M D_{r}$ are the values of the estimators $M D_{l}$ and $M D_{M L E}$ for $r$ replications and $M D$ is the value of the exponential distribution true median.

The Confidence Interval for the Exponential Distribution Median

Next, the confidence interval for the median of the exponential distribution, $M D$, is derived by modifying the confidence interval for the mean of the exponential distribution, $\mu$. Let $X_{1}, X_{2}, \ldots, X_{n}$ be a random sample of size $n$ from the exponential distribution with parameter $\lambda$, that is, $X \sim \operatorname{Exp}(1 / \lambda)$, then the exact two sided $100(1-\alpha) \%$ confidence interval for the exponential distribution mean, $\mu$, is given by (Trivedi, 2001):

$$
P\left(\frac{2 \sum_{i=1}^{n} X_{i}}{\chi_{(2 n, \alpha / 2)}^{2}}<\mu=\frac{1}{\lambda}<\frac{2 \sum_{i=1}^{n} X_{i}}{\chi_{(2 n, 1-\alpha / 2)}^{2}}\right)=1-\alpha
$$

where the $\chi_{(v, p)}^{2}$ is the $p^{\text {th }}$ percentile of a Chisquare distribution with $v$ degrees of freedom. The $\chi^{2}$-value can be read off from the $\chi^{2}$-table for the distribution (for example, see Kinney, 1997, page 506). Now, from equation (3) the exponential distribution median, $M D$, is given as follows:

$$
\mathrm{MD}=\frac{1}{\lambda} \ln (2)=\mu \ln (2) \Rightarrow \frac{\mathrm{MD}}{\ln (2)}=\frac{1}{\lambda}=\mu
$$

The $\mathrm{MD}_{\mathrm{MLE}}$ Confidence Interval

This confidence interval is obtained by substituting the result from (14) into equation (13); this gives the exact $100(1-\alpha) \%$ confidence interval for the exponential distribution median, $M D$, as follows: 


\section{ABU-SHAWIESH M.O.A}

$$
\begin{aligned}
\mathrm{P}\left(\frac{2 \sum_{\mathrm{i}=1}^{\mathrm{n}} \mathrm{X}_{\mathrm{i}}}{\chi_{(2 \mathrm{n}, \alpha / 2)}^{2}}\right. & \left.<\frac{\mathrm{MD}}{\ln (2)}=\frac{1}{\lambda}<\frac{2 \sum_{\mathrm{i}=1}^{\mathrm{n}} \mathrm{X}_{\mathrm{i}}}{\chi_{(2 \mathrm{n}, 1-\alpha / 2)}^{2}}\right) \\
& =\mathrm{P}\left(\frac{2 \ln (2) \sum_{\mathrm{i}=1}^{\mathrm{n}} \mathrm{X}_{\mathrm{i}}}{\chi_{(2 \mathrm{n}, \alpha / 2)}^{2}}<\mathrm{MD}<\frac{2 \ln (2) \sum_{\mathrm{i}=1}^{\mathrm{n}} \mathrm{X}_{\mathrm{i}}}{\chi_{(2 \mathrm{n}, 1-\alpha / 2)}^{2}}\right) \\
& =1-\alpha
\end{aligned}
$$

The $M D_{M L E}$ confidence interval is exact. It is based on the fact that $2 \lambda \sum_{i=1}^{n} X_{i}$ follows a $\chi_{(2 n)}^{2}$ distribution. The coverage probability must be exactly $95 \%$.

\section{The $\mathrm{MD}_{\mathrm{MD} 1}$ Confidence Interval}

This confidence interval is obtained by substituting the result from (10) into equation (13) to give the exact $100(1-\alpha) \%$ confidence interval for the exponential distribution median, $M D$, as follows:

$$
\begin{aligned}
\mathrm{P}\left(\frac{2\left(\mathrm{n} \mathrm{MD}_{1} / \ln (2)\right)}{\chi_{(2 \mathrm{n}, \alpha / 2)}^{2}}\right. & \left.<\frac{\mathrm{MD}}{\ln (2)}<\frac{2\left(\mathrm{n} \mathrm{MD}_{1} / \ln (2)\right)}{\chi_{(2 \mathrm{n}, 1-\alpha / 2)}^{2}}\right) \\
& =\mathrm{P}\left(\frac{2 \mathrm{n} \mathrm{MD}_{1}}{\chi_{(2 \mathrm{n}, \alpha / 2)}^{2}}<\mathrm{MD}<\frac{2 \mathrm{nMD}_{1}}{\chi_{(2 \mathrm{n}, 1-\alpha / 2)}^{2}}\right) \\
& =1-\alpha
\end{aligned}
$$

The $M D_{1}$ confidence interval is not exact. Its expression in (16) is based on equation (10) which is only an approximation of the statistics In order to see that, the performance of the $M D_{l}$ confidence interval is studied by calculating the coverage probability, the average width and the standard error using Monte-Carlo simulations. Actual, approximate and exact confidence intervals based on the sample median $M D_{1}$ can be also constructed using standard methods.
The Monte Carlo Simulation Study

A Monte Carlo simulation was designed to compare and study the behavior of the two estimators $M D_{l}$ and $M D_{M L E}$ and investigate the behavior of the proposed approximate confidence intervals for the exponential distribution median, MD. FORTRAN programs were used to generate the data from the exponential distribution and run the simulations and to make the necessary tables. Results are from the exponential distribution with parameter $\lambda$ which was set to 1 and 0.5 , to increase skewness. The more the repetition, the more accurate are simulated results, therefore 10,000 random samples of sizes $n=10,15,20,30,40$, 50 and 100 were generated.

Table (1) shows the simulated results for the root mean square error, RMSE, and the average of $M D_{1}$ 's and $M D_{\mathrm{MLE}}$ 's (AVG) to illustrate that both estimators are approximately unbiased for the true median of the exponential distribution, $M D$. The simulated results for the coverage probability $(\hat{\mathbf{P}})$, average width (AW) and standard error (SE) of the exact confidence interval for the exponential mean, $\mu$, and the two proposed approximate confidence intervals for the exponential distribution median, $M D$, are shown in tables (2-4). The criteria used to evaluate the exact and proposed approximate confidence intervals is the value of the coverage probability $(\hat{\mathbf{P}})$ and average width (AW); a good method should have an observed coverage probability $(\hat{\mathbf{P}})$ near to the nominal coverage probability and a small scaled average width (AW).

The simulation results in Table 1 show that the maximum likelihood estimator of the median, $M D_{M L E}$, is a much better estimator for the population median of the exponential distribution, $M D$, than the sample median, $M D_{l}$. While both estimators are approximately unbiased, the root mean square error, RMSE, for the sample median, $M D_{l}$, is larger than that of the maximum likelihood estimator of the median, $M D_{M L E}$. It should be noted that the accuracy of the maximum likelihood estimator of the median, $M D_{M L E}$, increases as the sample size, $n$, increases which clearly provides a very good estimator, even considering that the discrepancy of these two estimators is very small 


\section{ADJUSTED CONFIDENCE INTERVAL FOR EXPONENTIAL DISTRIBUTION MEDIAN}

-that is, these two estimators asymptotically coincide.

As shown in Tables 2-4, the simulation results show that the coverage probability $(\hat{\mathbf{P}})$ for the confidence interval of the mean and the approximate confidence interval of the median based on the MLE method for the exponential distribution are the same and very close to the nominal confidence coefficient.

The approximate confidence interval of the median based on the sample median method for the exponential distribution provides the lower coverage probability $(\hat{\mathbf{P}})$ and gives the lowest width among the three methods. The average widths (AW) for the two proposed confidence interval methods are approximately the same for moderate and large sample sizes. However, the estimated average width (AW) for the sample median method is the shortest among all considered methods, but it has poor coverage probability. Furthermore, as sample sizes increases, the performance of the proposed confidence interval based on the MLE method improves, but is still much lower than the nominal confidence.

Table 1: The Root Mean Square Error and Average of the Two Estimators for the Exponential Distribution Median

\begin{tabular}{|c|c|c|c|c|}
\hline \multirow{2}{*}{$\begin{array}{c}\text { Sample } \\
\text { Size (n) }\end{array}$} & \multicolumn{4}{|c|}{$\lambda=1$ (True Median $=0.69315)$} \\
\cline { 2 - 5 } & $\mathrm{RMSE}\left(M D_{l}\right)$ & $\mathrm{AVG}\left(M D_{l}\right)$ & $\mathrm{RMSE}\left(M D_{M L E}\right)$ & $\mathrm{AVG}\left(M D_{M L E}\right)$ \\
\hline 10 & 0.31575 & 0.74851 & 0.22369 & 0.69487 \\
\hline 15 & 0.26754 & 0.72676 & 0.18040 & 0.69332 \\
\hline 20 & 0.22459 & 0.72003 & 0.15715 & 0.69394 \\
\hline 30 & 0.18276 & 0.71180 & 0.12808 & 0.69335 \\
\hline 40 & 0.15907 & 0.70649 & 0.11106 & 0.69342 \\
\hline 50 & 0.14108 & 0.70459 & 0.09887 & 0.69439 \\
\hline 100 & 0.10005 & 0.69894 & 0.06961 & 0.69265 \\
\hline \multirow{2}{*}{$\begin{array}{c}\text { Sample } \\
\text { Size (n) }\end{array}$} & $\mathrm{RMSE}\left(M D_{l}\right)$ & $\mathrm{AVG}\left(M D_{1}\right)$ & $\mathrm{RMSE}\left(M D_{M L E}\right)$ & $\mathrm{AVG}\left(M D_{M L E}\right)$ \\
\hline 10 & 0.63150 & 1.49703 & 0.44739 & 1.38973 \\
\hline 15 & 0.53509 & 1.45352 & 0.36080 & 1.38663 \\
\hline 20 & 0.44918 & 1.44005 & 0.31430 & 1.38788 \\
\hline 30 & 0.36553 & 1.42359 & 0.25615 & 1.38671 \\
\hline 40 & 0.31814 & 1.41299 & 0.22211 & 1.38684 \\
\hline 50 & 0.28215 & 1.40917 & 0.19773 & 1.38877 \\
\hline 100 & 0.20010 & 1.39788 & 0.13922 & 1.38530 \\
\hline
\end{tabular}


Table 2: Coverage Probabilities, Average Width and Standard Error for the Confidence Interval of the Mean of the Exponential Distribution

\begin{tabular}{|c|c|c|c|c|c|c|}
\hline \multirow{3}{*}{$\mathrm{n}$} & \multicolumn{6}{|c|}{$1-\alpha=0.95$} \\
\hline & \multicolumn{3}{|c|}{$\lambda=1$} & \multicolumn{3}{|c|}{$\lambda=0.5$} \\
\hline & $\hat{\mathbf{P}}$ & AW & $\mathrm{SE}$ & $\hat{\mathbf{P}}$ & $\mathrm{AW}$ & $\mathrm{SE}$ \\
\hline 10 & 94.55 & 1.504 & 0.484 & 94.55 & 3.007 & 0.968 \\
\hline 15 & 94.84 & 1.148 & 0.299 & 94.84 & 2.297 & 0.598 \\
\hline 20 & 94.51 & 0.964 & 0.218 & 94.51 & 1.928 & 0.437 \\
\hline 30 & 94.58 & 0.762 & 0.141 & 94.58 & 1.524 & 0.282 \\
\hline 40 & 94.94 & 0.650 & 0.104 & 94.94 & 1.299 & 0.208 \\
\hline 50 & 94.79 & 0.576 & 0.082 & 94.79 & 1.153 & 0.164 \\
\hline 100 & 95.02 & 0.399 & 0.040 & 95.02 & 0.798 & 0.080 \\
\hline
\end{tabular}

Table 3: Coverage Probabilities, Average Width and Standard Error for the Confidence Interval of the Median of the Exponential Distribution with $\lambda=1$

\begin{tabular}{|c|c|c|c|c|c|c|}
\hline \multirow{3}{*}{$\mathrm{n}$} & \multicolumn{5}{|c|}{$1-\alpha=0.95$} \\
\cline { 2 - 7 } & \multicolumn{5}{|c|}{ MD $_{\text {MLE Method }}$} & \multicolumn{3}{c|}{ MD $_{\text {MD } 1}$ Method } \\
\cline { 2 - 7 } & $\hat{\mathbf{P}}$ & $\mathrm{AW}$ & $\mathrm{SE}$ & $\hat{\mathbf{P}}$ & $\mathrm{AW}$ & $\mathrm{SE}$ \\
\hline & 94.55 & 1.123 & 0.336 & 85.11 & 1.042 & 0.466 \\
\hline 10 & 94.84 & 0.834 & 0.207 & 82.76 & 0.796 & 0.305 \\
\hline 15 & 94.51 & 0.693 & 0.151 & 83.92 & 0.668 & 0.215 \\
\hline 30 & 94.58 & 0.542 & 0.098 & 83.41 & 0.528 & 0.139 \\
\hline 40 & 94.94 & 0.459 & 0.072 & 83.00 & 0.450 & 0.103 \\
\hline 50 & 94.79 & 0.405 & 0.057 & 83.28 & 0.400 & 0.081 \\
\hline 100 & 95.02 & 0.279 & 0.028 & 82.95 & 0.277 & 0.040 \\
\hline
\end{tabular}

Table 4: Coverage Probabilities, Average Width and Standard Error for the Confidence Interval of the Median of the Exponential Distribution with $\lambda=0.5$

\begin{tabular}{|c|c|c|c|c|c|c|}
\hline \multirow{3}{*}{$\mathrm{n}$} & \multicolumn{6}{|c|}{$1-\alpha=0.95$} \\
\cline { 2 - 7 } & \multicolumn{5}{|c|}{ Confidence Interval Method } \\
\cline { 2 - 7 } & $\hat{7}$ & $\mathrm{MD}$ MLE Method & $\hat{\mathbf{P}}$ & $\mathrm{AW}$ & $\mathrm{SE}$ \\
\cline { 2 - 7 } & $\hat{\mathbf{P}}$ & $\mathrm{AW}$ & 0.671 & 85.11 & 2.085 & 0.933 \\
\hline 10 & 94.55 & 2.246 & 0.414 & 82.76 & 1.592 & 0.609 \\
\hline 15 & 94.84 & 1.669 & 0.303 & 83.92 & 1.337 & 0.430 \\
\hline 20 & 94.51 & 1.387 & 0.195 & 83.41 & 1.056 & 0.277 \\
\hline 30 & 94.58 & 1.085 & 0.144 & 83.00 & 0.901 & 0.206 \\
\hline 40 & 94.94 & 0.918 & 0.114 & 83.28 & 0.799 & 0.162 \\
\hline 50 & 94.79 & 0.811 & 0.056 & 82.95 & 0.553 & 0.080 \\
\hline 100 & 95.02 & 0.558 & &
\end{tabular}




\section{ADJUSTED CONFIDENCE INTERVAL FOR EXPONENTIAL DISTRIBUTION MEDIAN}

Numerical Example

This example is taken from Wilk, et al. (1962); the data set represents the lifetimes (in weeks) of 34 transistors in an accelerated life test. The transistors were tested and the test continued until all of them failed. The lifetimes for the 34 transistors (in weeks) were recorded as follows:

$\begin{array}{rrrrrrrrr}3, & 4, & 5, & 6, & 6, & 7, & 8, & 8, & 9, \\ 9, & 9, & 10, & 10, & 11, & 11, & 11, & 13, & 13, \\ 13, & 13, & 13, & 17, & 17, & 19, & 19, & 25, & 29, \\ 33, & 42, & 42, & 52, & 52, & 52, & 52 & & \end{array}$

The sample mean $\bar{X}=18.912$ weeks, the exponential median $M D_{1}=13$ weeks, the exponential median $M D_{2}=13.108$ weeks and the skewness is 1.265695 , which is highly skewed distribution.

Furthermore, $\hat{\lambda}=\frac{1}{\bar{X}}=\frac{1}{18.912}=0.05287 \cong 0.053$ and using the approximation derived earlier - results in $\hat{\lambda}=\frac{\ln (2)}{M D_{1}}=\frac{0.69315}{13}=0.05332 \cong 0.053$ which indicates that the two values are very close and therefore the approximation is good. Based on Kibria (2006), the above data set are assumed to come from an exponential distribution with mean $\mu=21$ weeks; using the Kolmogorov-Smirnov test, the K-S statistic = 0.1603 and the $p$-value $=0.3125$, it indicates that the sample data are from an exponential distribution with mean $\mu=21$ weeks, and therefore (from equation (3)) has a median $M D$ $=14.556$ weeks. The resulting $95 \%$ confidence interval and the corresponding confidence width

Table 5: The 95\% Confidence Intervals for the

\begin{tabular}{|c|c|c|}
\multicolumn{4}{c}{ Lifetimes Data } \\
$\begin{array}{c}\text { Confidence } \\
\text { Interval Method }\end{array}$ & $\begin{array}{c}95 \% \text { Confidence } \\
\text { Interval }\end{array}$ & Width \\
\hline Exact for Mean & $(13.874,27.308)$ & 13.434 \\
\hline $\mathrm{MD}_{\mathrm{MLE}}$ & $(9.617,18.929)$ & 9.312 \\
\hline $\mathrm{MD}_{\mathrm{MD} 1}$ & $(9.537,18.772)$ & 9.235 \\
\hline
\end{tabular}

for the exact confidence interval for the exponential mean and the two proposed methods for the exponential median are calculated and given in table (5).

From table (5), it is observed that the exact confidence interval for the exponential mean, as expected, covered the hypothesized true population mean of $\mu=21$ weeks and also the proposed confidence intervals for the exponential median, $M D$, covered the hypothesized true population median $M D=$ 14.556 weeks. However, the proposed confidence interval for the exponential median, $M D$, based on the sample median, $M D_{1}$, provided the shortest confidence interval width.

\section{Conclusion}

The median - one of the most important and popular measures for location - has many good features. The median confidence interval is useful for one parameter families, such as the exponential distribution, and it may not need to be adjusted if censored observations are present. The maximum likelihood estimation is a popular statistical method used to make inferences about parameters of the underlying probability distribution from a given data set. This study proposed an approximate confidence interval for the median of the exponential distribution, $M D$, based on two estimators, the sample median, $M D_{1}$, and the maximum likelihood estimator of the median, $M D_{M L E}$.

The results of this study show that using a maximum likelihood estimator, $M L E$, for the population median of the exponential distribution, $M D$, is better alternative to the classical estimator based on the sample median, $M D_{1}$. As shown by the study results, the maximum likelihood estimator of the median, $M D_{M L E}$, provides a good estimation for the population median of the exponential distribution, $M D$, and the proposed confidence interval based on this estimator had a good coverage probabilities compared to the sample median method. However, it produced slightly wider estimated width. It appears that the sample size, $n$, has significant effect on the two proposed confidence interval methods. Moreover, both of the proposed methods are computationally simpler. If scientists and 


\section{ABU-SHAWIESH M.O.A}

researchers are conservative about the smaller width, they might consider confidence interval based on sample median method as a possible interval estimator for the population median of the exponential distribution, $M D$. Finally, the results obtained from the simulation study coincided with that of the numerical example.

\section{Acknowledgement}

The author would like to thank the Hashemite University for their cooperation during the preparation of this article.

\section{References}

Balakrishnan, N., \& Basu, A. P. (1996). The exponential distribution: theory, methods and applications, CRC press.

Bettely, G., Mettric, N., Sweeney, E., \& Wilson, D. (1994). Using statistics in industry: Quality improvement through total process control, ( $I^{\text {st }}$ Ed.). London: Prentice Hall International Ltd.

Casella, G., \& Berger, R. L. (2002). Statistical inference. Pacific Grove, CA: Duxbury.

Daniel, W. W. (1990). Applied nonparametric statistics $\left(2^{\text {nd }} E d\right.$.). Duxbury, Canada: Thomson Learning.

Francis, A. (1995). Business mathematics and statistics. London: DP Publications, Ltd.

Kececioglu, D. (2002). Reliability engineering handbook. Lancaster, UK: DEStech Publications.

Kibria, B. M. G. (2006). Modified confidence intervals for the mean of the asymmetric distribution. Pakestanian Journal of Statistics, 22(2), 109-120.

Kinney, J. (1997). Probability: An introduction with statistical applications. New York: John Wiley and Sons, Inc.

Lewis, E. E. (1996). Introduction to reliability engineering, $\left(2^{\text {nd }} E d\right.$.). New York: John Wiley and Sons, Inc.

Maguire, B. A., Pearson, E. S., \& Wynn, A. H. A. (1952). The time intervals between industrial accidents. Biometrika, 39, 168-180.

Montgomery, D. C. (2005). Introduction to statistical quality control $\left(5^{\text {th }} E d\right.$.). New York: John Wiley and Sons, Inc.
Patel, J. K., Kapadia, C. H., \& Owen, D. B. (1976). Handbook of statistical distributions. New York: Marcel Dekker.

Sinha, A., \& Bhattacharjee, S. (2004). Test of parameter of an exponential distribution in predictive approach. Pakestanian Journal of Statistics, 20(3), 409-414.

Smithson, M. (2001). Correct confidence intervals for various regression effect sizes and parameters: The importance of noncentral distributions in computing intervals. Educational and Psychological Measurement, 61, 605-532.

Trivedi, K. S. (2001). Probability and statistics with reliability, queuing, and computer science applications $\left(2^{\text {nd }} E d\right.$.). New York: John Wiley and Sons, Inc.

Van der Vaart, A.W., (1998). Asymptotic Statistics, 1-496. Cambridge University Press, Cambridge.

Wilk, M. B., Gnanadesikan, R., \& Huyett, M. J. (1962). Estimation of parameters of the gamma distribution using order statistics. Biometrika, 49, 525-545 